\title{
A Rare Case of Calcifying Pseudoneoplasms of the Neural Axis in Lumbar Spine
}

\author{
Venkatesan Ravi ${ }^{1}$ Pammi Srinath ${ }^{1, \odot}$ \\ ${ }^{1}$ Department of Spine Surgery, Apollo Hospitals, Chennai, India
}

\begin{abstract}
Address for correspondence Pammi Srinath, MBBS, DNB, Department of Spine Surgery, Counter No, 14, Apollo Hospitals, No. 21, Greams Road, off Greams Lane, Thousand Lights, Chennai 600006, India (e-mail: drsrinath.p@gmail.com).
\end{abstract}

\begin{abstract}
Keywords

- CAPNON

- non-neoplastic

- calcified mass
\end{abstract}

\section{Introduction}

Calcified pseudoneoplasm of the neural axis (CAPNON) is a very rare, non-neoplastic calcified pathological entity occurring anywhere in the central nervous system (CNS). Overall, less than 100 cases were reported, of which less than $50 \%$ were from the spine. ${ }^{1}$

They are also known as fibro-osseous lesions of the CNS. The exact etiology is still unknown, but it is believed to be reactive in nature. It was first described by Rhodes and Davis in 1978. ${ }^{2}$ CAPNON may occur in the spine and also intracranially, although incidence in the latter is more common. We present a case of CAPNON in the spine, considering clinical presentation, imaging, histopathology, differentials, treatment options, and outcome. These lesions are benign in nature and the symptoms are due to mass effect. It is important to distinguish them from more neoplastic lesions, as complete resection is curative.

\section{Case Report}

We present a case of a 53-year-old female with complaints of back pain, radiating to right lower limb, with

published online April 19, 2021
DOI https://doi.org/

10.1055/s-0040-1719221 ISSN 2277-954X. no neurological deficit or gait instability. The patient is a known case of rheumatoid arthritis with no previous history of trauma, tumor or infection. On examination, there was tenderness at the L4-L5 level, straight leg raising test was negative with power $5 / 5$ in both lower limbs, and deep tendon reflexes in the normal range.

MRI showed calcified intradural extramedullary mass, extending into the right lateral recess, which was isointense on T1- and T2-weighted sequences ( - Fig. 1A, B). Hemilaminectomy was done on the right side, mass was found around the dura, and adherent extending in to right L5 nerve root foramen. Mass was round and firm. Complete resection of the lesion was done. Histopathological examination showed multiple fragments of fibrocollagenous and degenerated tissue with foci of calcification, granular material surrounded by inflammatory cells composed of histiocytic aggregates and granuloma, and a giant cell reaction suggestive of calcified pseudoneoplasm of the neural axis ( - Fig. 2).

At 6 months follow-up, the patient is pain-free with no radicular symptoms. 

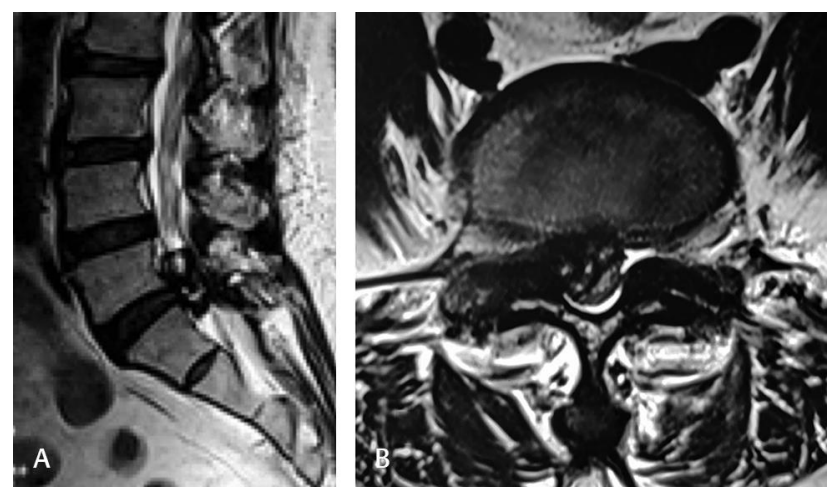

Fig. $1 \mathrm{MRI}$ of lumbar spine, T2-weighted image-sagittal section (A) and axial section (B) showing the calcified mass at L4-L5 level extending in to the right $L 5$ recess.

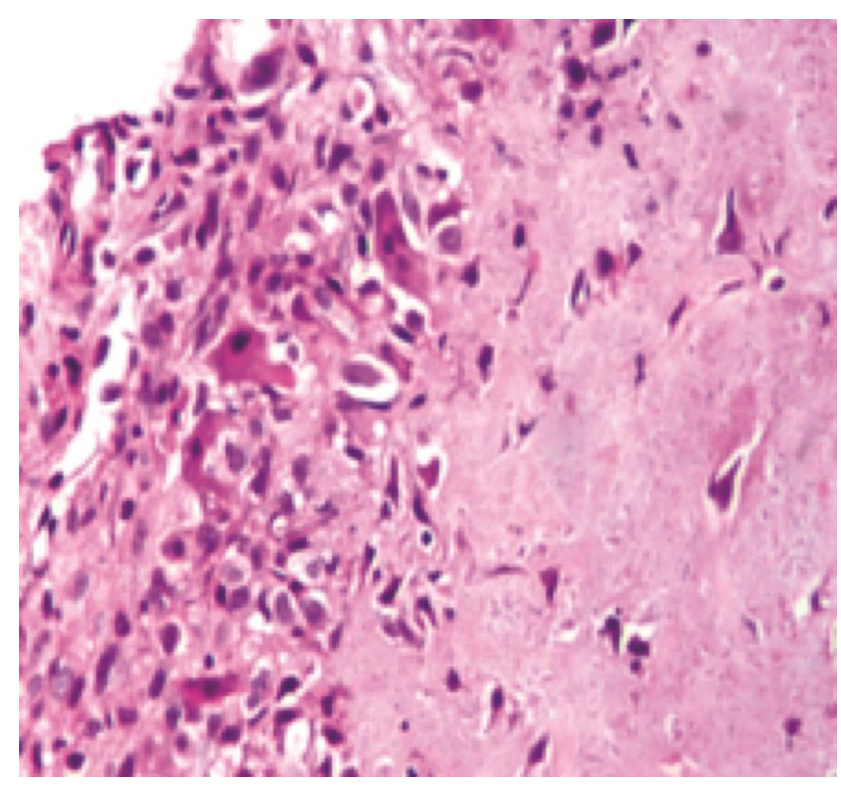

Fig. 2 Histopathological examination of epidural tissue from lumbar spine. Hematoxylin and eosin (H\&E) staining with magnification-400x, showing histiocytes and giant cells at the periphery of a chondromyxoid area.

\section{Discussion}

CAPNON is a lesion of the CNS. Although there is no age predominance, occurrence is more among the male population. $^{3-5}$ They are present with more prevalence in the brain than in the spine. Even in spine, presentation in lumbar spine is very rare. Symptoms in spine include local pain, or radiculopathy, and are usually due to mass effect. It is difficult to find on $\mathrm{X}$-rays. In CT scan, there is an appearance of calcification without bony erosion..$^{6-8}$ No contrast enhancement is reported. In MRI, lesions usually appear hypointense in both T1-weighted and T2-weighted sequences, but they may also present as isointense lesions. Contrast usually shows enhancement, which may be homogenous, nonhomogenous, or involve the lesion rim. ${ }^{1,7}$ Differential diagnosis may be a herniated calcified disc, meningioma, chordoma, schwannoma, or tuberculosis. ${ }^{3,9}$
Histopathologically, there is a chondromyxoid matrix with variable amounts of fibrous tissue, and calcification with foreign body type giant cells. ${ }^{3}$ A complete resection is the treatment. There are no recommendations for any chemotherapy or radiotherapy after excision. ${ }^{1}$

Recurrence in case of CAPNON is very unlikely. According to Stienen et al, ${ }^{1} 92.3 \%$ of patients with spinal CAPNON and $93.7 \%$ of patients with intracranial CAPNON showed no signs of recurrence. Although many studies recommend complete resection, in terms of recurrence, there was no significant difference between complete and incomplete resection. ${ }^{1}$

\section{Conclusion}

CAPNON in lumbar spine is a rare presentation. This entity should be taken as differential in cases of calcified intraspinal and intracranial lesions. Even though CT and MRI give a clue about the diagnosis, confirmation is only based on histopathology. Surgical resection is the treatment of choice.

\section{Conflict of Interest}

None declared.

\section{Acknowledgements}

The authors are thankful to Dr Ashok Parameswaran, Consultant Histopathology and Chief of Laboratory Services, Apollo Hospitals, Chennai for his insight and expertise with regard to the diagnosis of this case.

\section{References}

1 Stienen MN, Abdulazim A, Gautschi OP, Schneiderhan TM, Hildebrandt G, Lücke S. Calcifying pseudoneoplasms of the neuraxis (CAPNON): clinical features and therapeutic options. Acta Neurochir (Wien) 2013;155(1):9-17

2 Rhodes RH, Davis RL. An unusual fibro-osseous component in intracranial lesions. Hum Pathol 1978;9(3):309-319

3 Bertoni F, Unni KK, Dahlin DC, Beabout JW, Onofrio BM. Calcifying pseudoneoplasms of theneuralaxis.JNeurosurg 1990;72(1):42-48

4 Mohapatra I, Manish R, Mahadevan A, Prasad C, Sampath S, Shankar SK. Calcifying pseudoneoplasm (fibro osseous lesion) of neuraxis (CAPNON) - a case report. Clin Neuropathol 2010;29(4):223-226

5 Jun C, Burdick B. An unusual fibro-osseous lesion of the brain. Case report. J Neurosurg 1984;60(6):1308-1311

6 Garen PD, Powers JM, King JS. Perot PL Jr. Intracranial fibro-osseous lesion. Case report. J Neurosurg 1989;70(3): 475-477

7 Aiken AH, Akgun H, Tihan T, Barbaro N, Glastonbury C. Calcifying pseudoneoplasms of the neuraxis: CT, MR imaging, and histologic features. AJNR Am J Neuroradiol 2009;30(6): $1256-1260$

8 Liccardo G, Lunardi P, Menniti A, Floris R, Pastore FS, Fraioli B. Calcifying pseudo-tumor of the spine: description of a case and review of the literature. Eur Spine J 2003;12(5): 548-551

9 Moser FG, Tourje EJ, Pressman BD, Blinderman EE. Calcifying pseudotumor of the cervical spine. Am J Neuroradiol 1994; 15(3):580 